\title{
Comparing soil moisture anomalies from multiple independent sources over different regions across the globe
}

\author{
Carmelo Cammalleri, Jürgen V. Vogt, Bernard Bisselink, and Ad de Roo \\ European Commission, Joint Research Centre (JRC), Ispra, Italy \\ Correspondence: Carmelo Cammalleri (carmelo.cammalleri@ec.europa.eu)
}

Received: 3 April 2017 - Discussion started: 10 May 2017

Revised: 16 August 2017 - Accepted: 29 October 2017 - Published: 14 December 2017

\begin{abstract}
Agricultural drought events can affect large regions across the world, implying the need for a suitable global tool for an accurate monitoring of this phenomenon. Soil moisture anomalies are considered a good metric to capture the occurrence of agricultural drought events, and they have become an important component of several operational drought monitoring systems. In the framework of the JRC Global Drought Observatory (GDO, http://edo.jrc.ec.europa. $\mathrm{eu} / \mathrm{gdo} /$ ), the suitability of three datasets as possible representations of root zone soil moisture anomalies has been evaluated: (1) the soil moisture from the Lisflood distributed hydrological model (namely LIS), (2) the remotely sensed Land Surface Temperature data from the MODIS satellite (namely LST), and (3) the ESA Climate Change Initiative combined passive/active microwave skin soil moisture dataset (namely $\mathrm{CCI})$. Due to the independency of these three datasets, the triple collocation (TC) technique has been applied, aiming at quantifying the likely error associated with each dataset in comparison to the unknown true status of the system. TC analysis was performed on five macro-regions (namely North America, Europe, India, southern Africa and Australia) detected as suitable for the experiment, providing insight into the mutual relationship between these datasets as well as an assessment of the accuracy of each method. Even if no definitive statement on the spatial distribution of errors can be provided, a clear outcome of the TC analysis is the good performance of the remote sensing datasets, especially CCI, over dry regions such as Australia and southern Africa, whereas the outputs of LIS seem to be more reliable over areas that are well monitored through meteorological ground station networks, such as North America and Europe. In a global drought monitoring system, the results of the error analysis
\end{abstract}

are used to design a weighted-average ensemble system that exploits the advantages of each dataset.

\section{Introduction}

Drought is a recurring natural extreme, triggered by lower than normal rainfall, and often exacerbated by a strong evaporative demand due to high temperatures and strong winds. Drought events may occur in all climates and in most parts of the world, since drought is defined as a temporary deviation from the local normal condition. Due to the usually wide extension of the area of interest, drought affects millions of people across the globe each year (Wilhite, 2000).

On the basis of the economic and natural sectors impacted by this phenomenon, a drought event is usually classified as meteorological, agricultural and hydrological drought, depending on the persistence of the water deficit within the hydrological cycle. Of particular interest for this study are the agricultural (or ecosystem) drought events, defined as prolonged periods with drier than usual soils that negatively affect vegetation growth and crop production, and, as a consequence, human welfare (Dai, 2011).

Soil moisture is commonly seen as one of the most suitable variables for monitoring and quantifying the impact of water shortage on vegetated lands due to its effects on the terrestrial biosphere and the feedbacks into the atmospheric system. As a consequence, time-aggregated soil moisture anomalies (e.g., monthly) are included in numerous drought monitoring systems from regional to continental scales (i.e., European Drought Observatory, http://edo.jrc.ec.europa.eu; United States Drought Monitor, http://droughtmonitor.unl.edu; African Flood and Drought 
Monitor, http://hydrology.princeton.edu/adm/; among others).

In the context of drought monitoring, the soil moisture dynamic over large areas is usually modeled through either distributed hydrological models or land-surface schemes of climate models (Crow et al., 2012; Sheffield et al., 2004), as well as by thermal or passive/active microwave remote sensing-derived quantities (see, e.g., Anderson et al., 2007; Houborg et al., 2012; Mo et al., 2010). With regard to globalscale monitoring, remote sensing-based approaches have the advantage of intrinsic worldwide coverage. However, microwave sensors can explore only the first few centimeters of soil and are characterized by decreasing sensitivity with increasing vegetation coverage (Jackson, 2006). In the case of thermal data, the lack of coverage during cloudy conditions and the nontrivial connection between thermal and soil moisture signals (Price, 1980) are other limitations. By contrast, diagnostic models allow for continuous monitoring of soil moisture at the desired soil depths, but the accuracy of the data is constrained by uncertainties in the parameterization of soil hydrological characteristics, as well as by the actual availability of near-real-time reliable meteorological forcing data. Generally, the use of in situ observations for large area monitoring is limited, mainly due to the lack of long records, the sparseness of recording stations and the high spatial heterogeneity of soil moisture fields.

It follows that both satellite measurements and model predictions are subject to errors and uncertainties that need to be accounted for in their interpretation and application (Gruber et al., 2016). This also suggests that a monitoring system based on a single dataset is rarely capable of providing global reliable estimates, and a combination of different data sources is desirable in order to minimize the errors in the detection of drought events. Recently, Cammalleri et al. (2015) demonstrated the value of an ensemble of modeled soil moisture anomalies for drought monitoring over Europe, similarly to the findings of the US National Land Data Assimilation System (NLDAS) (Dirmeyer et al., 2006). However, a key point in combining different modeled data is the need to estimate the affinity and divergence between the models across the modeling domain.

In the most recent years, the triple collocation (TC) technique (Stoffelen, 1998) has been established as a practical approach to evaluate the unknown error variance (with respect to the truth) of three mutually independent measurement systems without knowing the "true" status of the system (Yilmaz and Crow, 2014). This technique has been widely applied in hydrology to estimate errors in soil moisture, as well as to evaluate precipitation and vegetation property indicators (Dorigo et al., 2010; McColl et al., 2014). One key requirement in TC is the existence of linearity between the three estimates and the truth, which can fail in the case of strongly seasonal geophysical variables such as soil moisture (Su et al., 2014). Luckily, drought monitoring systems are usually based on soil moisture anomalies rather than actual values, hence providing a partial remedy to this problem and making soil moisture anomalies directly suitable for this methodology (Miralles et al., 2010). However, since most TC studies focused on soil moisture dynamics rather than standardized anomalies, specific analyses are required to evaluate the accuracy of each dataset across the spatial domain.

In the framework of operational monitoring of agriculture and ecosystem drought, the availability of soil moisture, or proxy datasets available in near-real time, is crucial; within the Global Drought Observatory (GDO, http://edo.jrc.ec.europa.eu/gdo/), developed by the Joint Research Centre (JRC) of the European Commission, the soil moisture outputs of the Lisflood hydrological model and the Land Surface Temperature (LST) anomalies derived from the Moderate-Resolution Imaging Spectroradiometer (MODIS) onboard the Terra satellite have been detected as suitable datasets for near-real-time monitoring. In particular, Cammalleri and Vogt (2016) have highlighted how monthlyaverage LST anomalies represent the best proxy of soil moisture variations across different climates in Europe when compared to other LST-derived quantities.

As a third dataset for the TC analysis, the combined active/passive microwave soil moisture dataset produced by the European Space Agency (ESA) in the context of the Climate Change Initiative (CCI) is used; even if this dataset is not currently updated in near-real time, it represents a valuable reference dataset for a globally consistent time series of microwave-based soil moisture maps (also, near-real-time updating is foreseen in the framework of the Copernicus Climate Change Services).

The agreement between anomaly time series derived from these three products has not been fully investigated in the literature, especially on a global scale; hence, given the independency of the three sources of data (hydrological model, and thermal and microwave remote sensing) and the likely fulfillment of the main TC key hypothesis (i.e., independency between the errors of the three datasets), the TC approach seems suitable for quantifying the spatial distribution of the errors associated with each dataset.

Following these considerations, the overall goal of this study is two-fold. First, the agreement between the monthly anomalies of the three datasets is evaluated, in order to identify the macro-areas where reliable monitoring of soil moisture extreme conditions can be performed based on these three datasets that are available globally and suitable for use in a near-real-time monitoring system. Second, the TC analysis is performed over those macro-areas in order to quantify the spatial distribution of the expected random errors for each model compared to the unknown true status. The ultimate objective of the error analysis reported in this study is to provide information on the accuracy of the datasets that can be injected into a weighted-average ensemble procedure for near-real-time detection of the occurrence of ecosystem drought events, thus contributing to the future development 
of a robust agricultural drought monitoring index within the GDO system.

\section{Methods}

Drought events are commonly defined as prolonged periods during which a given drought indicator significantly deviates from the usual condition for the specific site and period (e.g., soil moisture content is lower than the climatology). Following this definition, this study will focus on standardized $z$ score values in order to make the different datasets directly comparable (i.e., minimizing the differences related to seasonality, soil depth, etc.). Specifically, monthly $z$-score values, or anomalies, are evaluated as

$z_{x, i, k}=\frac{x_{i, k}-\mu_{x, i}}{\sigma_{x, i}}$,

where $x_{i, k}$ is the monthly average variable for the $i$ th month at the $k$ th year, and $\mu_{x, i}$ and $\sigma_{x, i}$ are the long-term average and standard deviation of the variable $x$ for the $i$ th month, respectively. The baseline period adopted to compute the 12 $\mu$ and $\sigma$ monthly reference values should be $15-30$ years in order to ensure a stable benchmark. The three datasets used here, as described in the next section, are the root zone soil moisture data from the Lisflood model ( $x=$ LIS), the ESA Climate Change Initiative skin soil moisture microwave combined product $(x=\mathrm{CCI})$ and the thermal remote sensing derived Land Surface Temperature $(x=\mathrm{LST})$; in the case of LST data, the sign of the anomalies is reversed due to the expected inverse relationship between soil moisture and LST.

The monthly aggregation period is chosen to ensure statistical robustness of the computed anomalies, as well as to minimize the presence of missing data in the remote sensing datasets due to sub-optimal acquisition conditions (e.g., cloudy days for LST). The transition from daily data to monthly aggregated values also ensures a reduction in the likely discrepancies among the three datasets introduced by the differences in the explored soil depth, since the phase shift in time-aggregated quantities is usually less marked (Campbell and Norman, 1998). Additionally, the anomalies computed according to Eq. (1), characterized by a null average and a unitary standard deviation, allow for a direct comparison of the different datasets thanks to the removal of potential biases. In the particular case of a regression analysis between two standardized anomaly quantities, the Pearson correlation coefficient, $R$, represents not only a measure of the linear dependency of the two random variables, but also the slope of the linear relationship and a proxy of the difference and biases of the two datasets. In this respect, $R$ can be seen as a good synthetic descriptor of the relationship between two standardized $z$-score datasets. The statistical significance of the existence of a positive correlation can be evaluated by means of the Student's $t$ test (two-sided) by computing the $R$ value corresponding to a significance level $p=0.05$.
Analysis of the correlation among the datasets is interesting in the framework of the TC technique and its basic hypotheses. In TC, a first key hypothesis is the existence of linearity between the "true" status of the system and the three models; this is formally expressed as

$z_{x}=\alpha_{x}+\beta_{x} z_{\Theta}+\varepsilon_{x}$,

where $z_{\Theta}$ is the unknown true dataset of soil moisture anomalies, $\alpha_{x}$ and $\beta x$ are the systematic slope and bias parameters for the dataset $x$ with respect to the truth, and $\varepsilon_{x}$ is the additive zero-mean random noise. It follows that the absence of a statistically significant linear relation between all three models openly violates this hypothesis.

Other key underlying hypotheses of TC are the stationarity of both signals and errors, the independency between the errors and the signal (error orthogonality) and the independence between the errors of the three datasets (zerocross correlation) (Gruber et al., 2016). Finally, operational limitations regard the minimum sample size of each dataset, which is commonly assumed equal to 100 values (Scipal et al., 2008; Dorigo et al., 2010), even if some other authors suggest much larger sample sizes for a lower relative uncertainty (Zwieback et al., 2012).

Under these assumptions, Stoffelen (1998) proposed a formulation to estimate each model error variance, $\sigma_{\varepsilon x}^{2}$, based on a combination of the covariance between the datasets. In this approach, known as the covariance notation (Gruber et al., 2016), the error variance values are computed without a common (arbitrary) reference dataset as

$$
\begin{aligned}
& \sigma_{\varepsilon_{1}}^{2}=\sigma_{1}^{2}-\frac{\sigma_{12} \sigma_{13}}{\sigma_{23}}, \\
& \sigma_{\varepsilon_{2}}^{2}=\sigma_{2}^{2}-\frac{\sigma_{21} \sigma_{23}}{\sigma_{13}}, \\
& \sigma_{\varepsilon_{3}}^{2}=\sigma_{3}^{2}-\frac{\sigma_{31} \sigma_{32}}{\sigma_{12}},
\end{aligned}
$$

where, for the sake of simplicity, LIS, LST and CCI were renamed 1, 2 and 3, respectively. The first term on the righthand side of Eq. (3) represents the single model data variance, whereas the second term represents the so-called sensitivity of the model to variations in the true status, which is a function of the covariance terms between the three models. The advantage of this formulation is to directly estimate the unscaled error variances, which can (eventually) be scaled to a common data space, if needed.

In the case of the application of the covariance notation to standardized quantities (with zero mean and unitary standard deviation), the error variance values computed through Eq. (3) are expressed as dimensionless multiples of standard deviation, and a transformation to a common data space is not needed.

Different performance metrics can be derived from the covariance notation, including relative error variance metrics such as the fractional root-mean-squared error (fRMSE, 
Draper et al., 2013) and the correlation coefficient of each model with the underlying true signal (McColl et al., 2014). However, these metrics can be derived from each other by means of simple relationships (see Gruber et al., 2016) and they are analogous to the absolute error variance values in the case of $z$-scores that have known unitary dataset variance.

\section{Data and materials}

\subsection{Lisflood model soil moisture}

Root zone soil moisture dynamics are simulated by means of the Lisflood model (de Roo et al., 2000), a GIS-based distributed hydrological rainfall-runoff routing model designed to reproduce the main hydrological processes that occur in large and trans-national European river catchments. The model simulates all the main hydrological processes occurring in the land-atmosphere system, including infiltration, actual evapotranspiration, soil water redistribution in three sub-layers (surface, root zone and sub-soil), surface runoff rooting to the channel, and groundwater storage and transport (Burek et al., 2013).

Static maps used by the model are related to topography (i.e., digital elevation model, local drain direction, slope gradient, elevation range), land use (i.e., land use classes, forest fraction, fraction of urban area), soil (i.e., soil texture classes, soil depth), and channel geometry (i.e., channel gradient, Manning's roughness, bankfull channel depth, channel length, bottom width and side slope). Root zone depth is defined for each modeling cell on the basis of soil type and land use, where the soil-related hydraulic properties are obtained from the ISRIC $1 \mathrm{~km}$ SoilGrids database (Hengl et al., 2014), whereas topography data are obtained from the Hydrosheds database (Lehner et al., 2008).

Daily meteorological forcing maps are derived from the European Centre for Medium-range Weather Forecasts (ECMWF) data as spatially resampled and harmonized by the JRC Monitoring Agricultural ResourceS (MARS) group. The dataset includes daily average air temperature, potential evapotranspiration (for soil, water and reference surfaces) and total rainfall at $0.25^{\circ}$ spatial resolution, which were resampled on the model grid using the nearest neighbor algorithm.

The model run used in this study includes daily maps at $0.1^{\circ}$ resolution between 1989 and 2015; the grid domain of this dataset is used as a reference for the other two, whereas the baseline for the anomalies' computation is defined by the period 2001-2015 in order to match the LST data availability. Monthly data to be used in Eq. (1) are computed as a simple average of all the data available for each month, given that no gaps can be found in this dataset due to its continuous nature as a hydrological model. However, some areas were masked out due to the minimum or null temporal dynamic of soil moisture, such as Greenland and the Sahara.

\subsection{Land Surface Temperature dataset}

The use of the Land Surface Temperature (LST) anomalies as a proxy of soil moisture anomalies is based on the wellknown role of LST in the surface energy budget as a control factor for the partitioning between latent and sensible heat fluxes. In recent years, the existence of a connection between soil moisture and LST has been analyzed, mainly through the thermal inertia and the triangle methods (e.g., Carlson, 2007; Verstraeten et al., 2006), as well as by using LST as a direct proxy (see, e.g., Park et al., 2014; Srivastava et al., 2016). In a study over the pan-European domain, Cammalleri and Vogt (2016) have demonstrated the good agreement between monthly LST and LIS-based root zone soil moisture $z$-score values during summer time, where LST outperforms other LST-based indicators such as the day-night difference and the surface-air gradient.

Following these findings, this study adopts the dataset collected by the Moderate-Resolution Imaging Spectroradiometer (MODIS) sensor onboard the Terra satellite (http://terra.nasa.gov/about/terra-instruments/modis) as a source of monthly-scale long records of LST maps. In particular, the MOD11C3 monthly CMG (Climate Modelling Grid) LST product is used in this study, which is constituted by monthly composited and averaged temperature and emissivity maps at a spatial resolution of $0.05^{\circ}$ over a regular latitude-longitude grid; data for the period 2001-2015 are used, being the only fully completed years at the time of the analysis.

This monthly composite product is obtained as an average of the clear-sky data in the MOD11C1 products on the calendar days of the specific month, which are derived after re-projecting and re-sampling the MOD11B1 product. Details on the algorithm used to obtain the daily MOD11B1 maps can be found in Wan et al. (2002); in summary, a double screening procedure is applied, based on (i) the difference between the two independent LST estimates of the day-night algorithm (Wan and $\mathrm{Li}, 1997$ ) and the generalized split-window algorithm (Wan and Dozier, 1996), and (ii) the histogram of the difference between daytime and nighttime LSTs.

LST monthly maps were spatially co-registered to the Lisflood $0.1^{\circ}$ regular latitude-longitude grid by means of a simple average of the values within each cell, and anomaly maps were computed according to Eq. (1) by using only the data for which LST $>1{ }^{\circ} \mathrm{C}$; this threshold value (commonly used in snowmelt and snow-rainfall discrimination procedures; WMO, 1986) allows removal from the analysis of the data that are likely affected by snow or frost.

\subsection{Microwave combined dataset}

The ESA Climate Change Initiative (CCI) aims at developing a multi-satellite soil moisture dataset by combining data collected in both the past and present by passive and active mi- 
crowave instruments (Liu et al., 2012; Wagner et al., 2012). The current version of the dataset (v03.2) combines data from nine different sensors (SMMR, ERS-1/2, TMI, SSM/I, AMSR-E, ASCAT, WindSat, AMSR2 and SMOS) between 1978 and 2015.

Satellite-based microwave estimates of soil moisture are usually related to the first few centimeters of a soil column (i.e., skin layer), which is quite closely related to the soil moisture content in the root zone (Paulik et al., 2014), except for very dry conditions in sandy soils. Additionally, numerous validations against land-surface models have highlighted good performance across the globe, with notable exceptions over densely vegetated areas (e.g., Loew et al., 2013).

The algorithm adopted to merge the different data sources is the one developed by Liu et al. (2012), which is a threestep procedure that (i) merges the original passive microwave products, (ii) merges the original active microwave products, and (iii) blends the two merged products into a single final dataset. The merging procedure of passive datasets includes pixel-scale separation between seasonality and anomalies, rescaling of the data based on the piece-wise cumulative distribution function (CDF) and merging of the dataset using a common reference seasonality. For the active microwave instruments, the CDFs are directly used to rescale the data under the assumption that active datasets have an identical dynamic range, this mainly due to the limited overlap between datasets. The final blending of the two merged datasets is obtained by adopting a common resolution of approximately $25 \mathrm{~km}$ and daily frequency, as well as by using the GLDAS1-Noah model (ftp://agdisc.gsfc.nasa.gov/data/s4pa/) as a reference dataset for the CDF matching.

In this study, the daily blended dataset is spatially resampled to a $0.1^{\circ}$ regular latitude-longitude grid (the same used in Lisflood simulations) by means of the nearest neighbor algorithm, and successively aggregated to a monthly timescale by simply averaging the data (only if at least eight daily values were available in the specific month). Monthly average maps were converted into $z$-score maps by using the baseline period 2001-2015 (the time frame available for the LST dataset). Monthly aggregated $z$-score values of skin soil moisture are analyzed, jointly with the other two datasets, under the assumption that time-aggregation and normalization procedures minimize some of the discrepancies that are likely present between skin and root zone daily time series.

\section{Results and discussion}

\subsection{Linear regression analysis}

Considering the assumption of linearity between each one of the datasets and the unknown true status of the system in TC, a preliminary analysis of the linear correlation between the three anomaly products has been performed in order to detect the macro-areas where the TC procedure can be applied without violating this basic hypothesis. The correlation analysis was performed by using only the monthly anomalies that were available for all three datasets, with a sample size of at least 100 values (max sample size $=12$ months $\times 15$ years $=180$ ), and by defining a minimum correlation threshold $\left(R_{0.05}\right)$ that ensures a statistical significance of the linear relationship on the basis of the Student's $t$ test (at $p=0.05$ ).

The map in Fig. 1 reports in grey the areas where all three datasets are significantly linearly correlated according to the described criteria, representing the areas where the first basic hypothesis of the TC is not clearly violated. It is worth pointing out that some areas are excluded from the analysis by the lack of data in LIS (low temporal variability, as over Greenland and the Sahara), LST (due to the minimum temperature threshold or low temporal variability) or CCI (densely vegetated areas, such as the Amazon forest and the Congo basin). These results suggest focusing the successive detailed analyses on five macro-regions (denoted by the boxes in Fig. 1) that have consistent positive correlation values for all three datasets; these areas are named, from now on, (1) NA (North America, including the contiguous US and Mexico), (2) EU (southern and central Europe), (3) SA (southern countries of the African continent and Madagascar), (4) IN (Indian subcontinent), and (5) AU (Australia) ${ }^{1}$.

The correlation coefficient maps over those regions, obtained by inter-comparing the three datasets, are reported in Figs. 2 to 4, where the cells in red and yellow are the ones with negative or non-significant correlation, respectively, whereas the blue scale represents the cells with increasing significant linear correlation (from light to dark tones). The comparison between LIS and LST (Fig. 2) shows an overall good agreement between the two datasets, with only minor areas characterized by negative/non-significant correlation values; notably, low correlation values can be observed over the Great Lakes and Rocky Mountain areas in the US, over the Alps in Europe, and in northern Angola and the western Himalaya. Similar results can be observed in Fig. 3, where LIS and CCI datasets are compared; this comparison shows an increasing number of negative values in the western US, the Alps, and southern Turkey, but overall high correlation values across most of the five regions. Finally, the comparison between LST and CCI reported in Fig. 4 shows an increase in areas with low/non-significant correlation in the eastern and western US and both northeastern and southeastern Europe and the Alps, whereas high correlation values can be observed all over the other regions.

On average, the data in Table 1 summarize the results obtained for all the regions together, as well as for each region independently, showing how CCI and LST are the two

\footnotetext{
${ }^{1}$ Consider the countries and boundaries reported here as only indicative of the interested areas; they may not under any circumstances be regarded as stating an official position of the European Commission.
} 


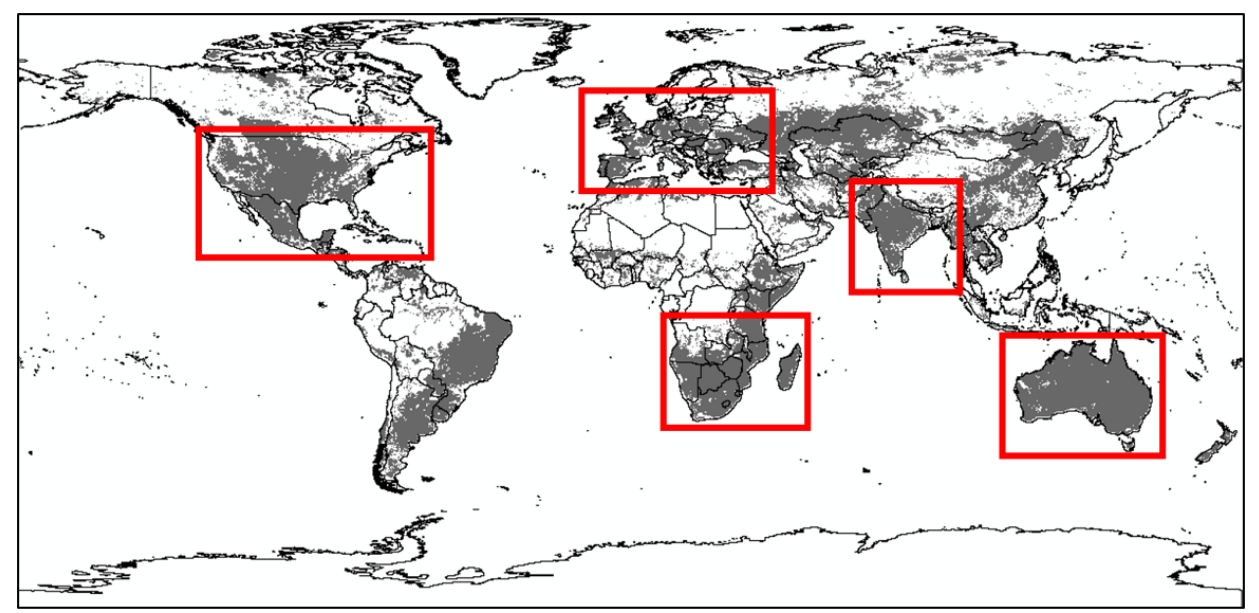

Figure 1. Map of the areas where all three models are positively significantly linearly correlated (cells in grey) according to the Student's $t$ test at $p=0.05$. The boxes delimitate the macro-regions selected for the successive analyses.

Table 1. Summary of the Pearson correlation coefficient values (average \pm standard deviation) observed for all the regions.

\begin{tabular}{lcccccc}
\hline Comparison & ALL & NA & EU & SA & IN & AU \\
\hline LIS vs. LST & $0.44 \pm 0.09$ & $0.41 \pm 0.08$ & $0.39 \pm 0.07$ & $0.48 \pm 0.09$ & $0.44 \pm 0.07$ & $0.50 \pm 0.10$ \\
LIS vs. CCI & $049 \pm 0.10$ & $0.47 \pm 0.09$ & $0.42 \pm 0.08$ & $0.48 \pm 0.10$ & $0.48 \pm 0.08$ & $0.58 \pm 0.11$ \\
CCI vs. LST & $0.56 \pm 0.13$ & $0.49 \pm 0.14$ & $0.37 \pm 0.09$ & $0.63 \pm 0.09$ & $0.52 \pm 0.10$ & $0.68 \pm 0.07$ \\
\hline
\end{tabular}

datasets best correlated with each other overall, even if this result is mainly driven by the results over the AU, SA and IN macro-areas. The LIS model data are similarly correlated with the ones of LST and CCI, with a more uniform distribution of the results across the various sub-regions. Another outcome of this analysis is that the area with the lowest average correlation between the three datasets is the EU, probably due to the high heterogeneity of this region at the $0.1^{\circ}$ spatial scale.

Some of the discrepancies observed in Figs. 2 to 4 can be explained by the differences in both horizontal and vertical resolution of the three raw datasets. LIS is characterized by a higher spatial resolution $(5 \mathrm{~km})$ compared to CCI $(25 \mathrm{~km})$ and a vertical resolution that encompasses the full root zone against the skin soil moisture of the latter; LST has a spatial resolution close to LIS but a vertical resolution that varies as a function of the vegetation coverage between skin (for bare soil) and root zone (for full vegetation coverage). The impact of such differences is partially reflected in the observed results, with CCI-LST better related over shallow soil in homogeneous areas, and LIS-LST better in agreement over sparse agricultural areas in Europe. Overall, it seems that the adopted expedients (i.e., monthly average, standardization) successfully minimized these issues, given that the results in Table 1 show a substantial and similar agreement of the three datasets in the main areas.

Additionally, the obtained results seem to suggest that it is reliable to adopt LST anomalies as a proxy of soil mois- ture anomalies, since there is a clear consistency of LST anomalies with the other two datasets. Similar results were obtained by Fang et al. (2016) over the continental United States, where the outputs of the thermal-based ALEXI (Atmosphere Land EXchange Inverse) model compare well with soil moisture anomalies from CCI and the Noah land-surface model. This consideration allows application of the TC analysis to the LST dataset as well, whereas most of the studies in the literature focus on land-surface modeled and microwave soil moisture datasets (i.e., Dorigo et al., 2010; Gruber et al., 2016; Su et al., 2014), with only a few notable exceptions including thermal data (e.g., Hain et al., 2011; Yilmaz et al., 2012).

\subsection{Triple collocation analysis}

The outcomes of the correlation analysis were used to detect the cells suitable for the TC technique; since a key hypothesis of the technique is the existence of a linear relation between each model and the (unknown) truth, a necessary condition (even if not sufficient) is the existence of linear relationships among the three datasets. As the outcome of the correlation analysis, around $10 \%$ of the five macro-areas were removed from the TC analysis due to the absence of this basic condition.

The maps in Figs. 5 to 7 show the main outcome of the TC analysis, which is the spatial distribution of the error variance (dimensionless, showing a multiple of the model standard de- 


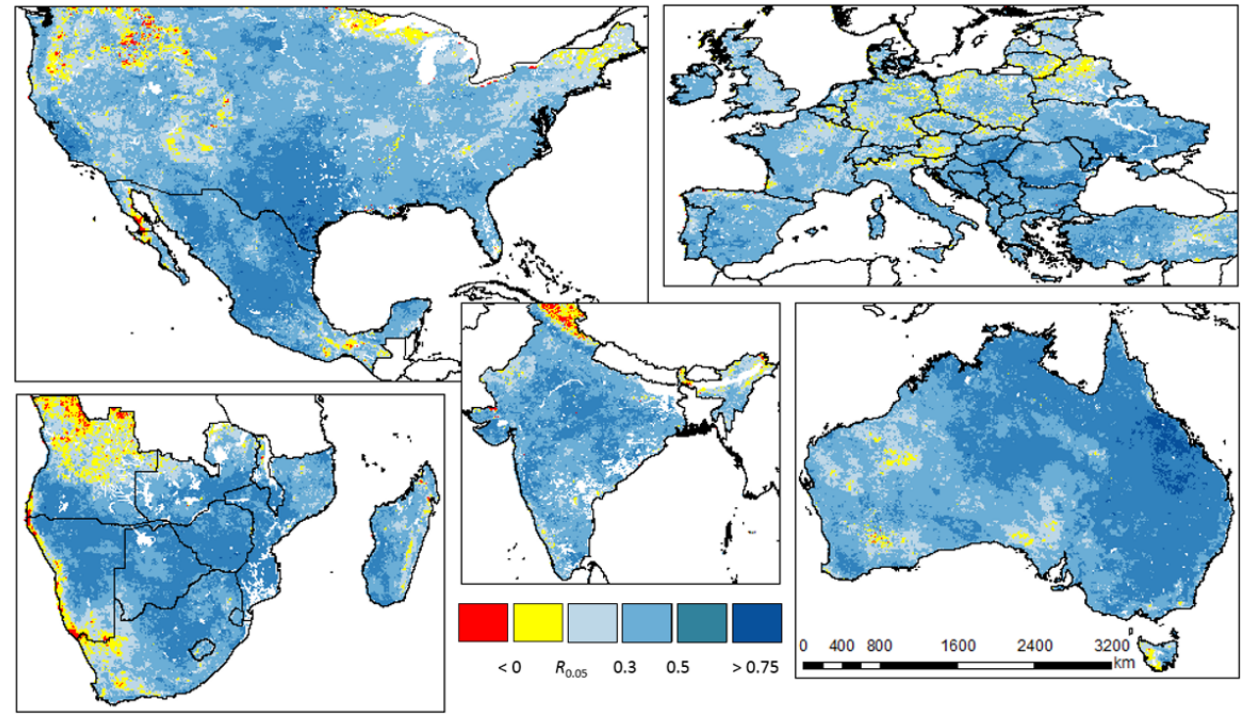

Figure 2. Spatial distribution of the Pearson correlation coefficient $(R)$ between Lisflood soil moisture anomalies (LIS) and land-surface temperature anomalies (LST) over the five selected macro-regions. Values in red and yellow are negatively correlated or not significant at $p=0.05$, respectively.

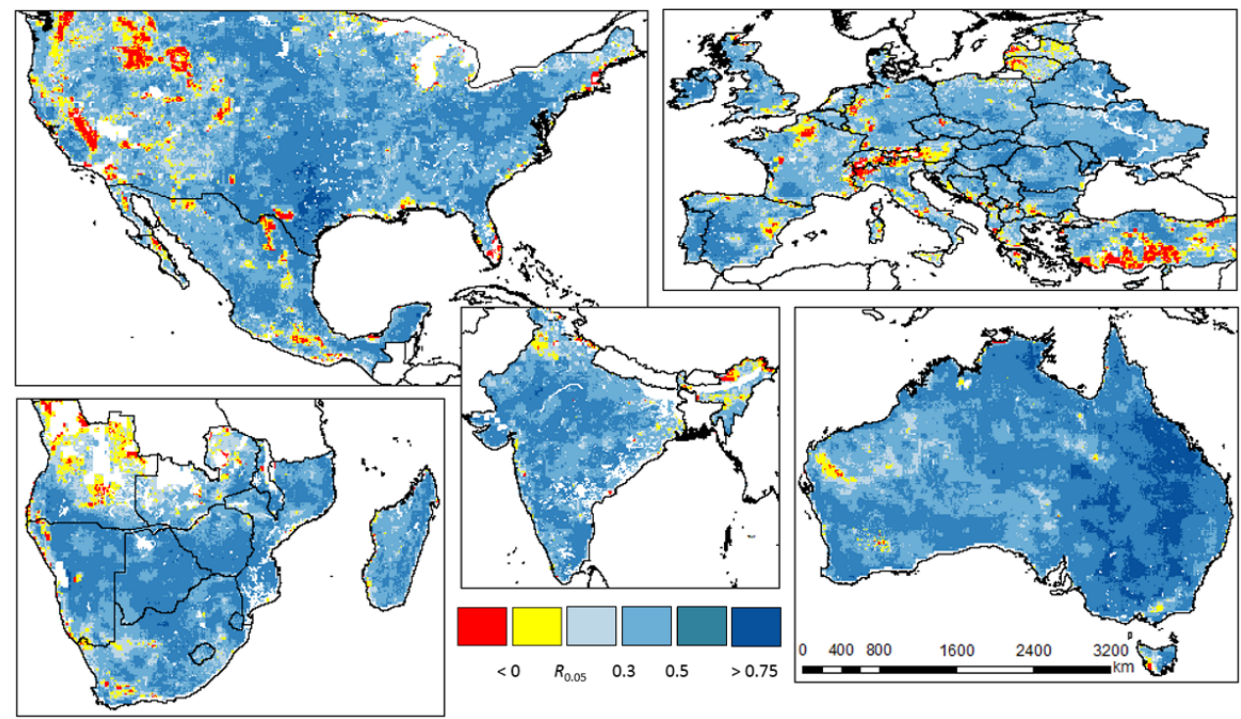

Figure 3. Spatial distribution of the Pearson correlation coefficient $(R)$ between Lisflood (LIS) and ESA Climate Change Initiative (CCI) soil moisture anomalies over the five selected macro-regions. Values in red and yellow are negatively correlated or not significant at $p=0.05$, respectively.

viation) for each model, as detailed by Eq. (3). The blank areas in those maps correspond to the cells where no significant linear correlation was observed between all three datasets. The results for LIS (Fig. 5) show that the highest errors are observed over the western US, the Northern Cape in South Africa and Western or Southern Australia, whereas the lowest errors are observed over the eastern US. On the opposite end, the LST dataset displays the highest errors over the latter area (Fig. 6), whereas the lowest errors are observed over Queensland in Australia, the Eastern Cape in South Africa and Lesotho. The maps in Fig. 7 show that the CCI dataset has consistent patterns of low error variance values over most of Australia, western India and the central US.

Overall, on the one hand, it seems evident that CCI tends to outperform the other two methods over dry areas such as Australia and southern Africa, but on the other hand, a region like the US is almost equally subdivided among the three datasets, where LIS performs better in the east, LST in the west and CCI in the center. Differences among products can be partially explained by the differences in the 


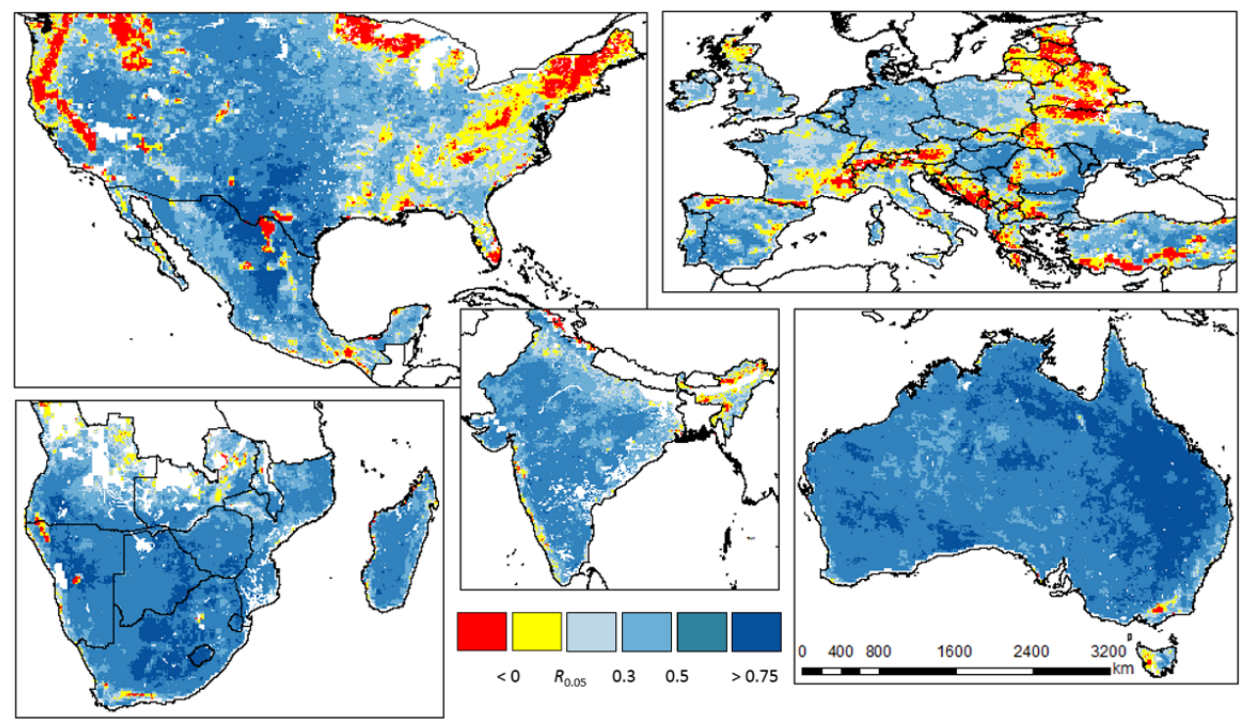

Figure 4. Spatial distribution of the Pearson correlation coefficient $(R)$ between ESA Climate Change Initiative soil moisture anomalies (CCI) and land surface temperature anomalies (LST) over the five selected macro-regions. Values in red and yellow are negatively correlated or not significant at $p=0.05$, respectively.

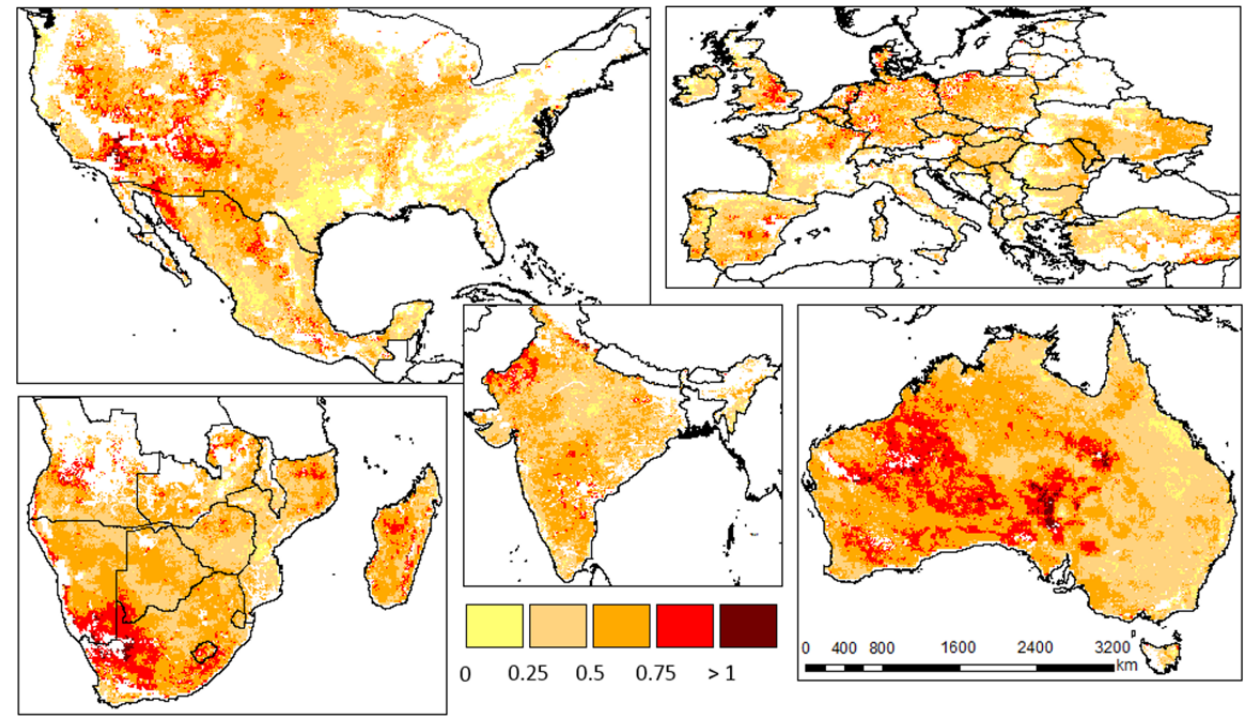

Figure 5. Spatial distribution of the error variance for the Lisflood (LIS) dataset over the five selected macro-regions.

soil layer monitored by each dataset; i.e., the microwave system captures the skin soil moisture, and Lisflood models the full root zone; indeed, even if the use of monthly anomalies allows minimization of some of the discrepancies, skin soil moisture remains more reliable for dry/bare areas (Das et al., 2015). Even if these considerations partially explain the agreement/disagreement of the three datasets, it is not straightforward to pinpoint in detail climate- and/or vegetation-derived patterns in the spatial distribution of the TC outputs.
These findings are summarized in the data reported in Table 2 , where the average error variance for each model and macro-area is reported beside its spatial standard deviation. The data in Table 2 confirm that CCI has an overall better performance (lower errors) than LIS and LST, which perform quite closely, mainly thanks to the very low error variance observed over Australia and, to a minor extent, southern Africa. The LIS model performs better over the NA and EU regions, likely due to the better meteorological forcing datasets available over those regions compared to the other macro-areas (due to denser ground networks). The LST dataset seems to 


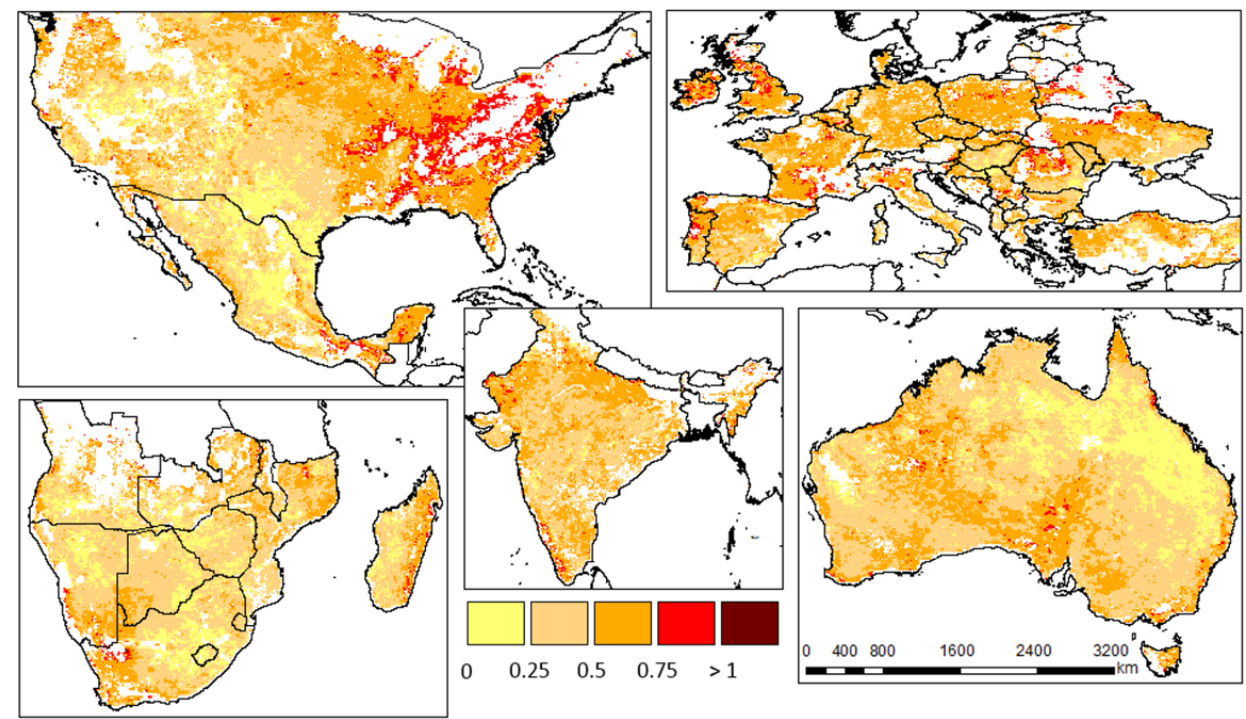

Figure 6. Spatial distribution of the error variance for the land surface temperature (LST) dataset over the five selected macro-regions.

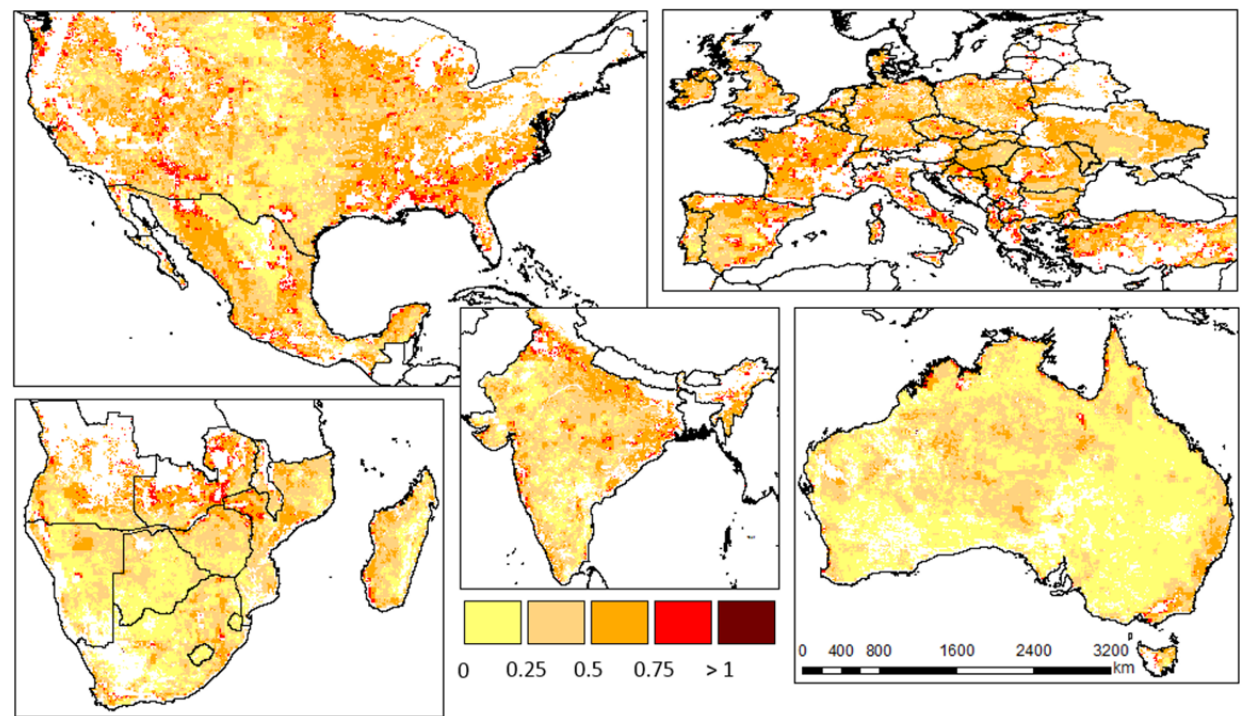

Figure 7. Spatial distribution of the error variance for the ESA Climate Change Initiative (CCI) dataset over the five selected macro-regions.

perform moderately well over all five macro-regions, with the only notable exception of EU; however, it rarely outperforms the other two datasets, constituting a "second-best" option in most of the cases. It is also worth pointing out that the CCI dataset is often masked out over those regions where the error of microwave techniques are likely high, whereas the data of the other two datasets are mostly produced globally; hence, a possible explanation of the better performance of CCI compared to LIS and LST may be linked to this preliminary screening of the data.

The outcome that LIS slightly outperforms the other two datasets over NA is in agreement with the results reported by Hain et al. (2011), where the Noah land-surface model slightly outperforms (on average) the microwave and thermal datasets over the contiguous US. However, it should be pointed out how the spatial distribution of the error estimates for LIS differs from the ones reported for Noah, likely due to the differences in both meteorological forcing and modeling approaches. Some qualitative analogies can also be observed with the results reported in Pierdicca et al. (2015), which show smaller average errors at a daily timescale over Europe for the ERA-LAND modeled datasets compared to two microwave-based datasets, even if both the temporal scale and the adopted methodology of the latter differ from the ones used in our study. These previous studies seem to confirm that land modeling approaches are more reliable, on av- 
Table 2. Summary of the TC error variance analysis, reporting the spatial average ( \pm standard deviation) values observed over each macroregion.

\begin{tabular}{lcccccc}
\hline Model & ALL & NA & EU & SA & IN & AU \\
\hline LIS & $0.48 \pm 0.13$ & $0.42 \pm 0.14$ & $0.44 \pm 0.12$ & $0.54 \pm 0.11$ & $0.49 \pm 0.10$ & $0.54 \pm 0.14$ \\
LST & $0.44 \pm 0.13$ & $0.46 \pm 0.15$ & $0.56 \pm 0.10$ & $0.37 \pm 0.10$ & $0.48 \pm 0.09$ & $0.38 \pm 0.11$ \\
CCI & $0.36 \pm 0.18$ & $0.46 \pm 0.16$ & $0.54 \pm 0.12$ & $0.30 \pm 0.14$ & $0.38 \pm 0.16$ & $0.17 \pm 0.10$ \\
\hline
\end{tabular}

erage, over these regions, likely due to the reliability of meteorological forcing and model parameterizations, even if there can be significant differences among the performances of different land-surface models.

Over the AU sub-region, the spatial distributions of the errors in CCI are quite in agreement with the results reported in Su et al. (2014) for two microwave datasets, with larger errors along the southeastern Australian coast. This result supports the assumption that microwave data are more reliable over dry bare soil areas, which is further highlighted by the results obtained in the SA and IN sub-regions. The subdivision of the NA domain into three main regions is similar to the one observed by Gruber et al. (2016) in comparing ASCAT and AMSR-E microwave datasets, suggesting key differences in the soil moisture behavior over these three sub-regions. Overall, the spatial patterns of microwave and land model errors show similarities to the ones observed by Dorigo et al. (2010), even if no thermal data were included in their analysis.

The error variance values can also be interpreted as the correlation coefficient of each dataset with the underlying true signal, following the definition of McColl et al. (2014). In fact, for the special case of anomalies with unitary variance $\left(\sigma_{x}^{2}=1\right)$, the TC-derived $R_{x}$ of each dataset is simply equal to $\sqrt{1 \sigma_{\varepsilon_{x}}^{2}}$, which ranges on average over all five regions (not shown) between 0.91 (for CCI in AU) and 0.66 (for LST over EU); these values show a good capability of the datasets to capture, on average, temporal variations in soil moisture anomalies.

\subsection{Insights for a weighted-average ensemble procedure}

In order to provide a simple synthetic representation of the likely best model for each area, the maps in Fig. 8 depict for each cell the dataset with the lowest error variance by associating different colors with the three datasets (red for LIS, blue for LST and green for CCI). Even if this approach is rather simplistic, as it cannot account for two products performing really closely over some areas, the major relevant features, like the predominance of the $\mathrm{CCI}$ model over Australia, are made evident by these maps.

The maps in Fig. 8 confirm CCI as the dataset with the lowest error variance values over most of AU, SA and IN, whereas the three datasets almost equally split the other two
Table 3. Fraction of each macro-area (as a percentage) where one model outperforms the other two.

\begin{tabular}{lrrrrrr}
\hline Model & ALL & NA & EU & SA & IN & AU \\
\hline LIS & 25.5 & 39.2 & 50.0 & 10.6 & 28.2 & 4.3 \\
LST & 25.7 & 28.8 & 23.1 & 36.0 & 20.3 & 18.6 \\
CCI & 48.8 & 32.0 & 26.9 & 53.4 & 51.5 & 77.1 \\
\hline
\end{tabular}

macro-areas; this is even more evident in the data reported in Table 3, where the percentage of sub-areas where each model is the best is reported. These data confirm the good performance of CCI over the AU, SA and IN macro-regions, whereas the NA territory is almost equally divided among the three datasets and LIS outperforms both LST and CCI over $50 \%$ of the EU domain. In the latter, the areas where the LIS dataset outperforms the other two datasets partially resemble the results obtained by Pierdicca et al. (2011) for the ERA-LAND model; however, the present study also includes remote sensing thermal data and not only microwave-derived datasets. Overall, the CCI dataset outperforms the other two datasets in about $50 \%$ of the cells, with the remainder almost equally split between LIS and LST.

Finally, the spatial distribution of the weighting factor of each dataset, computed according to the least square theory (Yilmaz et al., 2012), is represented in Figs. 9 to 11. The color scale of the figures was designed to represent in a neutral color the cells that have a weighting factor close to the one for a simple average $(1 / 3)$, in green scale the weights greater than a simple average (larger contribution) and in orange the weights lower than the simple average (smaller contribution). The visual intercomparison of these three maps further emphasizes the good performance of the CCI product over AU and SA, the best performance of LIS over the eastern US and EU, and the good results obtained for LST in the western US and northern AU. It is worth noting that the use of a weighted average based on the TC error analysis does not seem to bring advantages over large areas of the central US, EU and eastern IN, where the weighting factors are close to the ones for a simple arithmetic average. The behavior of the weighting factors over the five macro-areas can be synthetized by the frequency diagram in Fig. 12. This plot shows the high fraction of weighting factors $>0.4$ for the CCI dataset, representing a predominant contribution on the ensemble mean of this product over the others, whereas 




Figure 8. Maps representing the best performing (lowest error variance) dataset for each cell according to the TC analysis.

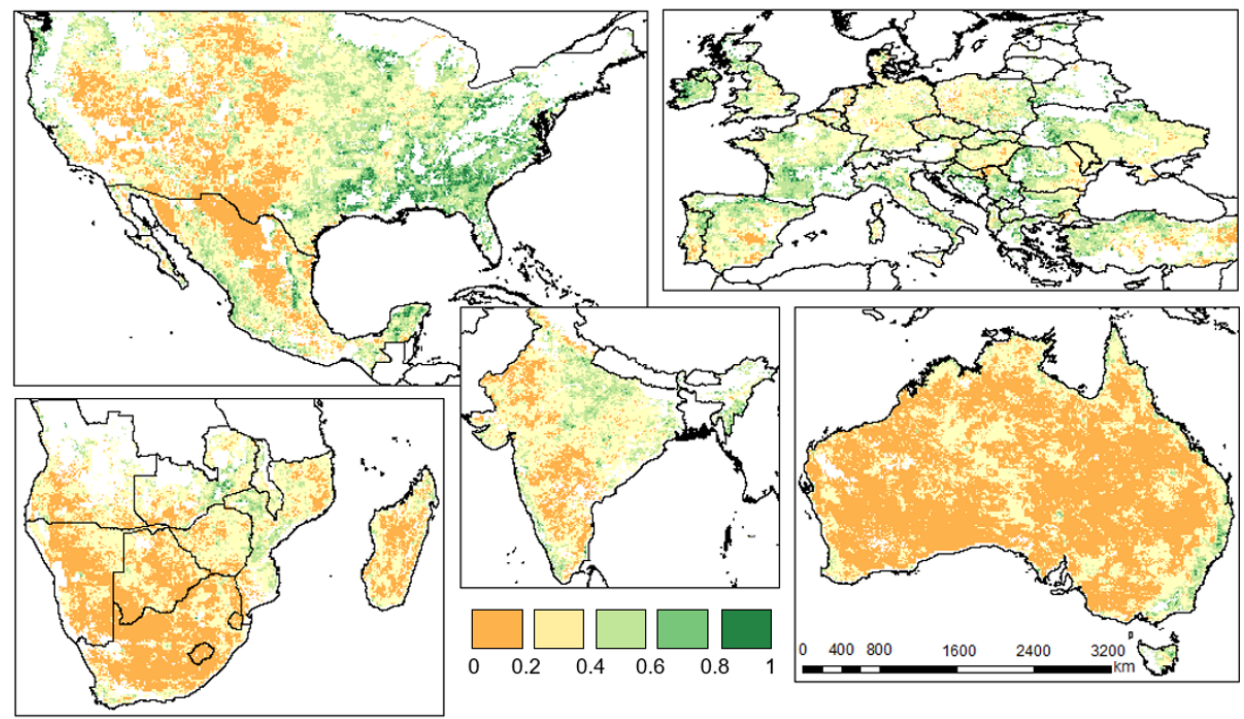

Figure 9. Maps representing the ensemble mean weighting factor for the LIS dataset according to the error maps derived from the TC analysis.

LST has a peak of frequency center around $1 / 3$ (arithmetic average) and LIS has a hint of a bi-modal distribution. These data, together with the maps in Fig. 8, confirm the fact that $\mathrm{CCI}$ outperforms the other two datasets in $50 \%$ of the domain, whereas LST is often the second-best option behind either CCI or LIS.

\section{Summary and conclusions}

Three datasets have been compared as a proxy of the unknown true status of soil moisture anomalies in the context of a global drought monitoring system under development by the JRC of the European Commission. The key assumption of the study is the inability of a single dataset to accurately capture the soil moisture dynamic over the large range of variability of conditions that can be observed at continental to global scale.

The inter-comparison between the three datasets, namely the outputs of the Lisflood hydrological model (LIS), MODIS-based land-surface temperature (LST) and the combined active/passive satellite microwave (CCI) data, confirms some inconsistencies between the three datasets over certain areas, as well as the difficulties in comparing the three datasets over specific areas (e.g., Sahara, Amazon rainfor- 


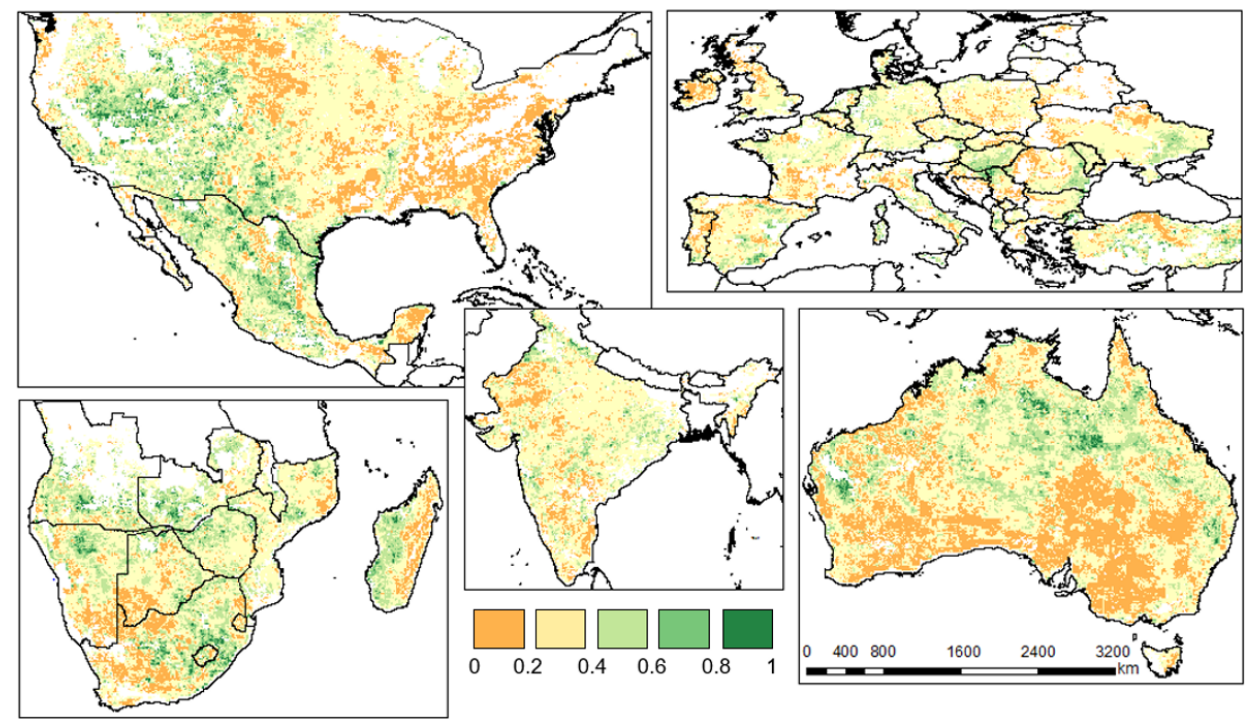

Figure 10. Maps representing the ensemble mean weighting factor for the LST dataset according to the error maps derived from the TC analysis.

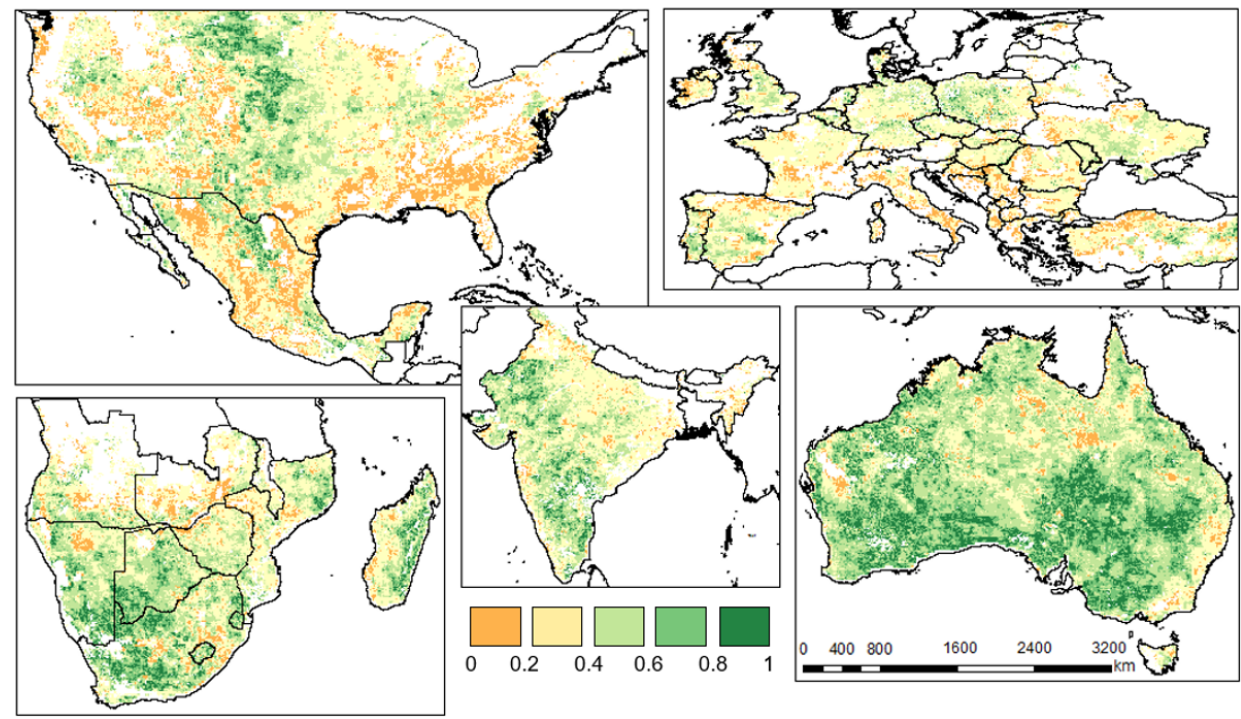

Figure 11. Maps representing the ensemble mean weighting factor for the CCI dataset according to the error maps derived from the TC analysis.

est) that are characterized by a lack of coverage from one or more datasets. Generally, the three datasets seem comparable over most of the globe, thanks to the use of time-aggregation and standardization procedures that remove temporal inconsistencies and biases among the series. Focusing the analysis only on the areas where the three datasets are substantially in agreement (following a linear regression analysis), five macro-regions were detected as suitable for further investigations according to the triple collocation (TC) technique. Under the hypothesis that certain criteria are met, the TC analysis allows quantification of the likely random error as- sociated with each model (with regard to the true status) even in the absence of an observation of the "truth".

The main outcome of the TC analysis further confirms the need for a multi-source approach for a reliable assessment of soil moisture anomalies over those five regions, given that no model outperforms the others (in terms of expected error variance) for the entire study domain. Emblematic are the results over North America, where each model outperforms the others in one sub-region, like the LIS approach in the eastern US, LST in the southwestern domain and CCI in the central US. Even if no clear insight into the general patterns of 


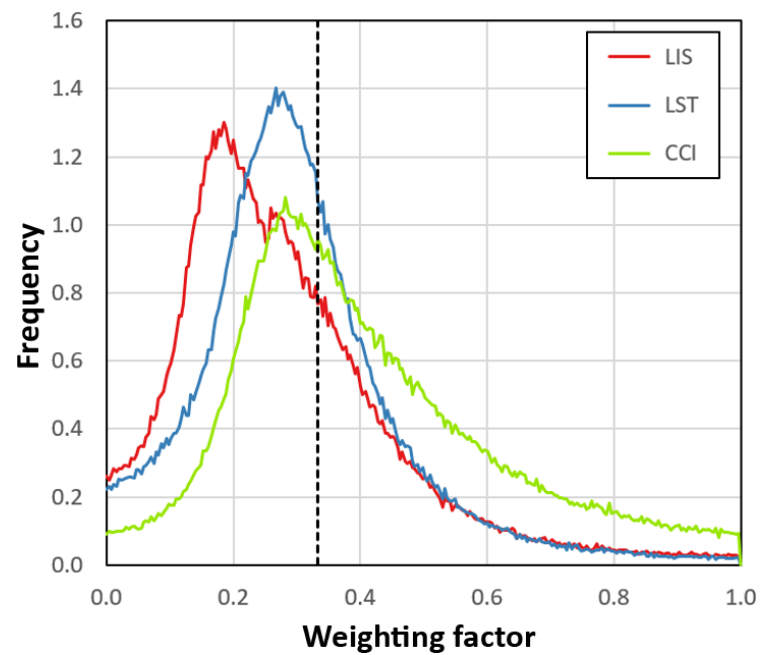

Figure 12. Frequency distribution of the ensemble mean weighting factor for each dataset computed according to the TC analysis. The black dotted line represents the value corresponding to a simple arithmetic average $(1 / 3)$.

the errors can be provided as an outcome of the study, overall, the obtained results seem to suggest that remote sensing datasets perform better over dry areas and sparsely monitored areas (e.g., Australia and southern Africa), whereas the LIS dataset seems more reliable over NA and EU, where dense networks of meteorological ground stations are deployed.

It has been highlighted how some differences among the datasets can also be related to the depth of the soil layer monitored by each dataset, i.e., the microwave system capturing the skin soil moisture, whereas Lisflood models the full root zone; indeed, even if the use of monthly anomalies allows minimization of some of the discrepancies and biases, our results confirm that skin soil moisture remains more reliable for areas where the effects of vegetation coverage are minimal (Das et al., 2015), whereas hydrological models are more suited for agricultural and densely vegetated regions. However, the three datasets seem to be overall comparable in terms of average performances, supporting the success of the adopted homogenization procedures. Some analogies between the obtained results and the ones already available in the literature have been found, but the inclusion of thermal data in the analysis enlarges the understanding of the mutual relationship between the different datasets.

The results of this study represent a robust starting point for the development of a global drought monitoring system based on such anomaly datasets, which can exploit the main findings of the TC analysis in order to develop a suitable ensemble product over the investigated regions. The error characterization derived from TC was used to estimate the weighing factors of an ensemble mean procedure, based on the least squares framework reported in Yilmaz et al. (2012). Currently, an operational implementation of such an ensem- ble product is foreseen for the GDO system as soon as the CCI product becomes available in near-real time.

Further analyses are required to be able to extend the test to the areas currently not included in this study, especially the ones where the three datasets are available but provide inconsistent or contrasting results. In this context, the analysis of further global datasets may help to unveil the reasons behind such discrepancies.

Data availability. The MARS meteorological dataset can be accessed at http://agri4cast.jrc.ec.europa.eu (European Commission JRC, 2017). NASA MODIS LST data can be accessed at https: //e4ft101.cr.usgs.gov/MOLT/MOD11C3.006/ (NASA, 2017). CCI data can be accessed at http://www.esa-soilmoisture-cci.org (ESA, 2017).

Competing interests. The authors declare that they have no conflict of interest.

Acknowledgements. The authors would like to thank the EC-JRC MARS group for providing the meteorological forcing, as well as NASA and ESA for making their satellite products publicly available.

Edited by: Kerstin Stahl

Reviewed by: two anonymous referees

\section{References}

Anderson, M. C., Norman, J. M., Mecikalski, J. R., Otkin, J. P., and Kustas, W. P.: A climatological study of evapotranspiration and moisture stress across the continental U.S. based on thermal remote sensing: II. Surface moisture climatology, J. Geophys. Res., 112, D11112, https://doi.org/10.1029/2006JD007507, 2007.

Burek, P., van der Knijff, J. M., and de Roo, A.: LISFLOOD: Distributed Water Balance and Flood Simulation Model, JRC Scientific and Technical Reports, EUR 26162 EN, 142 pp., https://doi.org/10.2788/24719, 2013.

Cammalleri, C. and Vogt, J. V.: On the role of Land Surface Temperature as proxy of soil moisture status for drought monitoring in Europe, Remote Sens., 7, 16849-16864, 2016.

Cammalleri, C., Micale, F., and Vogt, J. V.: On the value of combining different modelled soil moisture products for European drought monitoring, J. Hydrol., 525, 547-558, 2015.

Campbell, G. S. and Norman, J. M.: An introduction to environmental biophysics, Springer-Verlag, New York (NY), USA https://doi.org/10.1007/978-1-4612-1626-1, 1998.

Carlson, T.: An overview of the "Triangle Method" for estimating surface evapotranspiration and soil moisture from satellite imagery, Sensors, 7, 1612-1629, 2007.

Crow, W. T., Kumar, S. V., and Bolten, J. D.: On the utility of land surface models for agricultural drought monitoring, Hydrol. Earth Syst. Sci., 16, 3451-3460, https://doi.org/10.5194/hess-163451-2012, 2012. 
Dai, A.: Drought under global warming: A review, Wiley Interdiscip. Rev. Clim. Change, 2, 45-65, 2011.

Das, K., Paul, P. K., and Dobesova, Z.: Present status of soil moisture estimation by microwave remote sensing, Cogent Geoscience, 1, 1084669, https://doi.org/10.1080/23312041.2015.1084669, 2015.

de Roo, A., Wesseling, C., and van Deusen, W.: Physically based river basin modelling within a GIS: The LISFLOOD model, Hydrol. Process., 14, 1981-1992, 2000.

Dirmeyer, P. A., Gao, X., Zhao, M., Guo, Z., Oki, T., and Hanasaki, N.: GSWP-2: multimodel analysis and implications for our perception of the land surface, B. Am. Meteorol. Soc., 87, 13811397, 2006.

Dorigo, W. A., Scipal, K., Parinussa, R. M., Liu, Y. Y., Wagner, W., de Jeu, R. A. M., and Naeimi, V.: Error characterisation of global active and passive microwave soil moisture datasets, Hydrol. Earth Syst. Sci., 14, 2605-2616, https://doi.org/10.5194/hess-142605-2010, 2010.

Draper, C., Reichle, R., de Jeu, R., Naeimi, V., Parinussa, R., and Wagner, W.: Estimating root mean square errors in remotely sensed soil moisture over continental scale domains, Remote Sens. Environ., 137, 288-298, 2013.

ESA: Climate Change Initiative Soil Moisture Project v03.2, available at: http://www.esa-soilmoisture-cci.org, last access: September 2017.

European Commission JRC: MARS Gridded Agro-meteorological data, available at: http://agri4cast.jrc.ec.europa.eu, last access: December 2017.

Fang, L., Hain, C. R., Zhan, X., and Anderson, M. C.: An intercomparison of soil moisture data products from satellite remote sensing and a land surface model, Int. J. Appl. Earth Obs., 48, 37-50, 2016.

Gruber, A., Su, C.-H., Zwieback, S., Crow, W., Dorigo, W., and Wagner, W.: Recent advances in (soil moisture) triple collocation analysis, Int. J. Appl. Earth Obs., 45, 200-211, 2016.

Hain, C. R., Crow, W. T., Mecikalski, J. R., Anderson, M. C., and Holmes, T.: An intercomparison of available soil moisture estimates from thermal infrared and passive microwave remote sensing and land surface modeling, J. Geophys. Res., 116, D15107, https://doi.org/10.1029/2011JD015633, 2011.

Hengl, T., de Jesus, J. M., MacMillan, R. A., Batjes, N. H., Heuvelink, G. B. M., Ribeiro, E., Samuel-Rosa, A., Kempen, B., Leenaars, J. G. B., Walsh, M. G., and Ruiperez Gonzalez, M.: SoilGrids1km - Global Soil Information Based on Automated Mapping, PLoS ONE, 9, e105992, https://doi.org/10.1371/journal.pone.0105992, 2014.

Houborg, R., Rodell, M., Li, B., Reichle, R., and Zaitchik, B.: Drought indicators based on model assimilated GRACE terrestrial water storage observations, Water Resour. Res., 48, W07525, https://doi.org/10.1029/2011WR011291, 2012.

Jackson, T. J.: Estimation of Surface Soil Moisture Using Microwave Sensors, Encyclopedia of Hydrological Sciences, Remote Sensing, 5, 54, https://doi.org/10.1002/0470848944.hsa060, 2006.

Lehner, B., Verdin, K., and Jarvis, A.: New global hydrography derived from spaceborne elevation data, Eos, 89, 93-94, 2008.

Liu, Y. Y., Dorigo, W. A., Parinussa, R. M., de Jeu, R. A. M., Wagner, W., McCabe, M. F., Evans, J. P., and van Dijk, A. I. J. M.:
Trend-preserving blending of passive and active microwave soil moisture retrievals, Remote Sens. Environ., 123, 280-297, 2012.

Loew, A., Stacke, T., Dorigo, W., de Jeu, R., and Hagemann, S.: Potential and limitations of multidecadal satellite soil moisture observations for selected climate model evaluation studies, Hydrol. Earth Syst. Sci., 17, 3523-3542, https://doi.org/10.5194/hess-173523-2013, 2013.

McColl, K. A., Vogelzang, J., Konings, A. G., Entekhabi, D., Piles, M., and Stoffelen, A.: Extended triple collocation: Estimating errors and correlation coefficients with respect to an unknown target, Geophys. Res. Lett., 41, 6229-6236, 2014.

Miralles, D. G., Crow, W. T., and Cosh, M. H.: Estimating spatial sampling errors in coarse-scale soil moisture estimates derived from point-scale observations, J. Hydrometeorol., 11, 14231429, 2010.

Mo, K. C., Long, L. N., Xia, Y., Yang, S. K., Schemm, J. E., and Ek, M. B.: Drought indices based on the Climate Forecast System Reanalysis and ensemble NLDAS, J. Hydrometeorol., 12, 185210, 2010.

NASA: MODIS MOD15A2H Collection 6, available at: https:// e4ft101.cr.usgs.gov/MOLT/MOD11C3.006/, last access: December 2017.

Park, J.-Y., Ahn, S.-R., Hwang, S.-J., Jang, C.-H., Park, G.-A., and Kim, S.-J.: Evaluation of MODIS NDVI and LST for indicating soil moisture of forest areas based on SWAT modeling, Paddy Water Environ., 12, 77-88, 2014.

Paulik, C., Dorigo, W., Wagner, W., and Kidd, R.: Validation of the ASCAT Soil Water Index using in situ data from the International Soil Moisture Network, Int. J. Appl. Earth Obs., 30, 1-8, 2014.

Pierdicca, N., Fascetti, F., Pulvirenti, L., Crapolicchio, R., and Munõz-Sabater, J.: Analysis of ASCAT, SMOS, in-situ and land model soil moisture as a regionalized variable over Europe and North Africa, Remote Sens. Environ., 170, 280-289, 2015.

Price, J. C.: The potential of remotely sensed thermal infrared data to infer surface soil moisture and evaporation, Water Resour. Res., 16, 787-795, 1980.

Scipal, K., Holmes, T., de Jeu, R., Naemi, V., and Wagner, W.: A possible solution for the problem of estimating the error structure of global soil moisture datasets, Geophys. Res. Lett., 35, L24404, https://doi.org/10.1029/2008GL035599, 2008.

Sheffield, J., Goteti, G., Wen, F., and Wood, E. F.: A simulated soil moisture based drought analysis for the United States, J. Geophys. Res., 109, D24108, https://doi.org/10.1029/2004JD005182, 2004.

Stoffelen, A.: Toward the true near-surface wind speed: Error modelling and calibration using triple collocation, J. Geophys. Res., 103, 7755-7766, 1998.

Srivastava, P. K., Islam, T., Singh, S. K., Gupta, M., Petropoulos, G. P., Gupta, D. K., Wan Jaafar, W. Z., and Prasad, R.: Soil moisture deficit estimation through SMOS soil moisture and MODIS land surface temperature, in: Satellite Soil Moisture Retrieval: Techniques and Applications, edietd by: Srivastava, P. K., Petropoulos, G. P., and Kerr, Y. H., Elsevier B.V., 2016.

Su, C.-H., Ryu, D., Crow, W. T., and Western, A. W.: Beyond triple collocation: Applications to soil moisture monitoring, J. Geophys. Res.-Atmos., 119, 6419-6439, 2014.

Verstraeten, W. W., Veroustraete, F., van der Sande, C. J., Grootaers, I., and Feyen, J.: Soil moisture retrieval using thermal inertia, de- 
termined with visible and thermal spaceborne data, validated for European forests, Remote Sens. Environ., 101, 299-314, 2006.

Wagner, W., Dorigo, W., de Jeu, R., Fernandez, D., Benveniste, J., Haas, E., and Ertl, M.: Fusion of active and passive microwave observations to create an essential Climate Variable data record on soil moisture. ISPRS Annal of the Photogrammetry, Remote Sensing and Spatial Information Sciences, volume I-7. XXII ISPRS Congress, 25 August-1 September 2012, Melbourne, Australia, 2012.

Wan, Z. and Dozier, J.: A generalized split-window algorithm for retrieving land surface temperature from space, IEEE T. Geosci. Remote Sens., 34, 892-905, 1996.

Wan, Z. and Li, Z.-L.: A physics-based algorithm for retrieving land-surface emissivity and temperature from EOS/MODIS data, IEEE T. Geosci. Remote Sens., 35, 980-996, 1997.

Wan, Z., Zhang, Y., Zhang, Q., and Li, Z.-L.: Validation of the landsurface temperature products retrieved from Terra Moderate Resolution Imaging Spectroradiometer data, Remote Sens. Environ., 83, 163-180, 2002.
Wilhite, D. A.: Drought as a natural hazard: Concepts and definitions. N: Disasters series, Routledge Publishers, UK, 213-230, 2000.

World Meteorological Organization: Intercomparison of models of snowmelt runoff, Operational Hydrological Report, 23, 1986.

Yilmaz, M. T. and Crow, W. T.: Evaluation of assumptions in soil moisture triple collocation analysis, J. Hydrometeorol., 15, 1293-1302, 2014.

Yilmaz, M. T., Crow, W. T., Anderson, M. C., and Hain, C. R.: An objective methodology for merging satellite- and modelbased soil moisture products, Water Resour. Res., 48, W11502, https://doi.org/10.1029/2011WR011682, 2012.

Zwieback, S., Scipal, K., Dorigo, W., and Wagner, W.: Structural and statistical properties of the collocation technique for error characterization, Nonlin. Processes Geophys., 19, 69-80, https://doi.org/10.5194/npg-19-69-2012, 2012. 